\title{
Novel monosaccharide fermentation products in Caldicellulosiruptor saccharolyticus identified using NMR spectroscopy
}

\author{
Nancy G Isern', Junfeng Xue ${ }^{3}$, Jaya V Rao ${ }^{3}$, John R Cort $^{2^{*}}$ and Birgitte K Ahring ${ }^{3 *}$
}

\begin{abstract}
Background: Caldicellulosiruptor saccharolyticus is a thermophilic, Gram-positive, non-spore forming, strictly anaerobic bacterium of interest in potential industrial applications, including the production of biofuels such as hydrogen or ethanol from lignocellulosic biomass through fermentation. High-resolution, solution-state nuclear magnetic resonance (NMR) spectroscopy is a useful method for the identification and quantification of metabolites that result from growth on different substrates. NMR allows facile resolution of isomeric (identical mass) constituents and does not destroy the sample.

Results: Profiles of metabolites produced by the thermophilic cellulose-degrading bacterium Caldicellulosiruptor saccharolyticus DSM 8903 strain following growth on different monosaccharides (D-glucose, D-mannose, L-arabinose, D-arabinose, D-xylose, L-fucose, and D-fucose) as carbon sources revealed several unexpected fermentation products, suggesting novel metabolic capacities and unexplored metabolic pathways in this organism. Both ${ }^{1} \mathrm{H}$ and ${ }^{13} \mathrm{C}$ nuclear magnetic resonance (NMR) spectroscopy were used to determine intracellular and extracellular metabolite profiles. One dimensional ${ }^{1} \mathrm{H}$ NMR spectral analysis was performed by curve fitting against spectral libraries provided in the Chenomx software; 2-D homonuclear and heteronuclear NMR experiments were conducted to further reduce uncertainties due to unassigned, overlapping, or poorly-resolved peaks. In addition to expected metabolites such as acetate, lactate, glycerol, and ethanol, several novel fermentation products were identified: ethylene glycol (from growth on D-arabinose), acetoin and 2,3-butanediol (from growth on D-glucose, L-arabinose, and D-xylose), and hydroxyacetone (from growth on D-mannose, L-arabinose, and D-xylose). Production of ethylene glycol from D-arabinose was particularly notable, with around $10 \%$ of the substrate carbon converted into this uncommon fermentation product.

Conclusions: The present research shows that C. saccharolyticus, already of substantial interest due to its capability for biological ethanol and hydrogen production, has further metabolic potential for production of higher molecular weight compounds, such as acetoin and 2,3-butanediol, as well as hydroxyacetone and the uncommon fermentation product ethylene glycol. In addition, application of nuclear magnetic resonance (NMR) spectroscopy facilitates identification of novel metabolites, which is instrumental for production of desirable bioproducts from biomass through microbial fermentation.
\end{abstract}

Keywords: Caldicellulosiruptor saccharolyticus, Nuclear magnetic resonance, Bioproducts, Acetoin, 2,3-Butanediol, Ethylene glycol

\footnotetext{
* Correspondence: john.cort@pnnl.gov; bka@tricity.wsu.edu

${ }^{2}$ Fundamental and Computational Sciences Directorate, Pacific Northwest

National Laboratory, PO Box 999, MSIN: K8-98, Richland, WA 99352, USA

${ }^{3}$ Center for Bioproducts and Bioenergy, Washington State University, 2710

Crimson Way, Richland, WA 99354, USA

Full list of author information is available at the end of the article
} 


\section{Background}

Caldicellulosiruptor saccharolyticus is a thermophilic, Gram-positive, non-spore forming, strictly anaerobic bacterium of interest in potential industrial applications, including the production of biofuels such as hydrogen or ethanol from lignocellulosic biomass through fermentation [1-6]. C. saccharolyticus has a broad substrate range, and can grow on a variety of simple (e.g., glucose, mannose, xylose, and arabinose) or complex (e.g. cellulose, hemicelluloses) carbohydrates that are often associated with lignocellulosic biomass [7]. C. saccharolyticus produces native cellulases and hemicellulases, enabling it to efficiently hydrolyze complex carbohydrates and use the released monosaccharides as carbon and energy sources. In addition, $C$. saccharolyticus can grow on biomass that is either pretreated (e.g., Miscanthus hydrolysate, sugar beet juice, and paper sludge) or untreated (e.g. wheat straw, pine wood, and bagasse) [6]. Furthermore, C. saccharolyticus is able to co-ferment different monosaccharides, such as glucose and xylose, without exhibiting carbon catabolite repression $[4,5]$, and it grows at high temperatures (optimum $65^{\circ} \mathrm{C}-70^{\circ} \mathrm{C}$ ) and tolerates a broad temperature range.

Because C. saccharolyticus appears well-suited for production of biofuels, its metabolism has been extensively studied. Aside from hydrogen, acetate is the major fermentation product, and lactate and ethanol are also produced by mixed fermentation pathways $[2,3]$. C. saccharolyticus has been reported to exhibit increased production of lactate, a more reduced end-product, during the transition to stationary phase, which coincides with a drastic decrease in glucose consumption and acetate production [8]. Previous work has shown that the Embden-Meyerhof (EM) pathway is the main route for glycolysis in $C$. saccharolyticus, with a theoretical yield of 4 moles of $\mathrm{H}_{2}$ and 2 moles of acetate per mole of glucose [3]. Analysis of the genome sequence reveals the presence of all of the EMpathway enzymes [4]. No evidence has been found for the presence of the Entner-Doudoroff (ED) and oxidative pentose phosphate pathways in C. saccharolyticus $[3,4]$.

Identification of metabolites can provide insight into metabolic pathway utilization [9]. High-resolution, solutionstate nuclear magnetic resonance (NMR) spectroscopy is a useful method for studying the changes in concentrations and fluxes of metabolites that result from growth on different substrates. NMR allows facile resolution of isomeric (identical mass) constituents and does not destroy the sample. To characterize the monosaccharide metabolism of $C$. saccharolyticus with the aim of characterizing its full metabolic potential for production of bioproducts, the present study used 1-D ${ }^{1} \mathrm{H}$-detected NMR spectroscopy together with resonance peak assignment and curve fitting for metabolite identification and quantification. Deconvolution and curve fitting in the Chenomx software package $[10,11]$ have previously been used to determine metabolite profiles in a variety of microbial metabolomics applications [12-15], and these methods were utilized for the purposes of this study. To confirm the identification and for manual assignment of unidentified metabolites, $1-\mathrm{D}{ }^{13} \mathrm{C}$-detected and 2-D homo- and heteronuclear NMR techniques were used to analyze the metabolic profiles of $C$. saccharolyticus grown on various monosaccharides. Several novel fermentation products were identified and quantified, indicating novel metabolic capacities that are not predicted in the current understanding of metabolism implied by the genome of this thermophilic bacterium and suggesting new potentials for use of this organism in production of bioproducts from cellulosic biomass.

\section{Results and discussion}

Peak assignment using the Chenomx software

Metabolite profile analyses of the culture supernatants and extracts were compared with spectra of uninoculated growth medium to identify compounds produced and secreted by $C$. saccharolyticus. Across all of our studies, approximately 50 metabolites were identified and quantified using spectral deconvolution and library-based assignment routines in the Chenomx 7.61 software (Table 1, Table 2, and Additional file 1). Approximately twenty spectral features, many of them having low intensity, remained unassigned. However, two of the more prominent unassigned features were assigned using 2-D NMR spectroscopy to 2,3-butanediol and hydroxyacetone. These assignments were confirmed by comparison to prepared standards, and approximate concentrations were estimated using spectral deconvolution to estimate peak areas. The major reduced fermentation products of interest produced from growth on each monosaccharide are summarized in Table 2 . Products such as amino acids that were also components of the growth media are not included, though studies with ${ }^{13} \mathrm{C}$-labeled glucose showed evidence for incorporation of ${ }^{13} \mathrm{C}$ into some amino acids, notably alanine and glycine. All concentrations were determined using Chenomx except those so noted in Table 2. We have determined that concentrations of major metabolites quantified using Chenomx are accurate to within a few percent of the measured value for any particular sample (data not shown).

\section{Metabolite coverage of NMR analysis}

A complete list of compound assignments is provided in the supplemental material (Additional file 1). Selected identifications and quantifications of specific metabolites illustrating differences in supernatant and cell extracts are shown in Figure 1. Observed ${ }^{1} \mathrm{H}$ and ${ }^{13} \mathrm{C}$ chemical shifts and corresponding assignments for major metabolites are shown in Table 1. Multiplicities in 1-D ${ }^{1} \mathrm{H}$ 
Table 1 Observed ${ }^{1} \mathrm{H}$ and ${ }^{13} \mathrm{C}$ chemical shifts and corresponding assignments for major metabolites

\begin{tabular}{|c|c|c|c|c|c|c|}
\hline Compound & Chemical shifts & & & & & \\
\hline acetate & $184.1(\mathrm{C} 1)$ & $1.90(\mathrm{H} 2)$ & $25.9(\mathrm{C} 2)$ & & & \\
\hline acetoin (3-hydroxybutanone) & $2.21(\mathrm{H} 1)$ & $27.8(\mathrm{C} 1)$ & $218.0(C 2)$ & $75.6(C 3)$ & $1.37(\mathrm{H} 4)$ & $21.1(C 4)$ \\
\hline (RR/SS) 2,3-butanediol & $1.14(\mathrm{H} 1)$ & $20.4(C 1)$ & $3.61(\mathrm{H} 2)$ & $74.3(C 2)$ & & \\
\hline ethanol & $3.66(\mathrm{H} 1)$ & $60.3(\mathrm{C} 1)$ & $1.17(\mathrm{H} 2)$ & 19.6 (C2) & & \\
\hline ethylene glycol & $3.66(\mathrm{H} 1)$ & $65.3(\mathrm{C} 1)$ & & & & \\
\hline glycerol & $3.65(\mathrm{H} 1 \mathrm{a})$ & $3.55(\mathrm{H} 1 \mathrm{~b})$ & $65.2(\mathrm{C} 1)$ & $3.78(\mathrm{H} 2)$ & 74.8 (C2) & \\
\hline hydroxyacetone (1-hydroxypropanone) & $4.37(\mathrm{H} 1)$ & $70.0(\mathrm{C} 1)$ & $215.3(C 2)$ & $2.15(H 3)$ & $27.2(\mathrm{C} 3)$ & \\
\hline lactate & $185.2(\mathrm{C} 1)$ & $4.11(\mathrm{H} 2)$ & $70.8(\mathrm{C} 2)$ & $1.33(\mathrm{H} 3)$ & $22.7(\mathrm{C} 3)$ & \\
\hline propylene glycol (1,2-propanediol) & $3.54(\mathrm{H} 1 \mathrm{a}) 3.43(\mathrm{H} 1 \mathrm{~b})$ & $69.3(C 1)$ & $3.87(\mathrm{H} 2)$ & $70.6(C 2)$ & $1.13(\mathrm{H} 3)$ & $20.7(C 3)$ \\
\hline
\end{tabular}

Multiplicities in 1-D ${ }^{1} \mathrm{H}$ spectra and observation of the expected cross peaks in 2-D COSY, HSQC, and HMBC spectra confirmed these assignments. All shifts are in agreement with expected and previously reported values.

spectra and observation of the expected cross peaks in 2-D ${ }^{1} \mathrm{H}-{ }^{1} \mathrm{H}$ COSY (COrrelation SpectroscopY), ${ }^{1} \mathrm{H}-{ }^{13} \mathrm{C}$ HSQC (Heteronuclear Single Quantum Coherence), and ${ }^{1} \mathrm{H}_{-}{ }^{13} \mathrm{C}$ HMBC (Heteronuclear Multiple Bond Correlation) spectra confirmed these assignments. All shifts are in agreement with expected and previously reported values.

\section{Identification of novel metabolites}

Acetate and lactate were the most abundant products during growth on each substrate, and small quantities of ethanol and glycerol were found in all cultures. In addition to acetate, lactate, glycerol, and ethanol, culture supernatants from growths on D-glucose also contained small amounts of the $\mathrm{C}_{4}$ compounds acetoin and 2,3butanediol (Table 2). Culture supernatants from cells grown on $\mathrm{D}$-arabinose contained a significant concentration $\left(\sim 23 \mathrm{mM} / \mathrm{OD}_{600}\right)$ of ethylene glycol, an uncommon fermentation product. Because ethylene glycol has single ${ }^{1} \mathrm{H}$ and ${ }^{13} \mathrm{C}$ chemical shifts, and because these shifts are nearly coincident with those of one of the $\mathrm{H} 5$ protons and C5 in one of the D-arabinose anomers, the assignment suggested by the Chenomx software was confirmed by spiking samples of uninoculated media and culture supernatant with ethylene glycol and observing the expected increase in intensity of the putative ethylene glycol peak in the HSQC spectrum (Figure 2). According to our confirmatory HPLC analysis, C. saccharolyticus produced the highest concentrations of ethylene glycol during late log phase (Figure 3). Although the ethylene glycol concentration seems slightly higher in the stationary phase compared to the late log phase (Figure 3 ), the difference is not statistically significant ( $\mathrm{P}=0.55$ using $t$-test, $\mathrm{n}=3)$. Propylene glycol was further observed (up to $5 \mathrm{mM}$ with longer growth time) in both supernatants and extracts from cultures grown on L-fucose.

Hydroxyacetone and 2,3-butanediol were not identified in our primary 1-D ${ }^{1} \mathrm{H}$ NMR screen using the Chenomx software, because its library does not contain their NMR spectra. Instead, these compounds were identified in supernatant mixtures after 2-D COSY, HSQC, and HMBC experiments were conducted to identify the unassigned features in the 1-D spectra. A representative HSQC spectrum with assignments for a mannose culture supernatant is shown in Figure 4. Identified compound concentrations were estimated by using spectral deconvolution to determine peak areas in $1-\mathrm{D}{ }^{1} \mathrm{H}$ spectra,

Table 2 Major fermentation products in the batch culture supernatants as a function of sugar substrate

\begin{tabular}{|c|c|c|c|c|c|c|c|c|c|}
\hline Monosaccharide & Acetate $^{a}$ & Lactate $^{a}$ & Ethanol & Ethylene glycol & 1,2-propanediol ${ }^{b}$ & Glycerol $^{a}$ & Acetoin $^{c}$ & 2,3-butanediol ${ }^{b}$ & Hydroxyacetone $^{b}$ \\
\hline D-glucose (Glu) & 37.2 & 1.4 & 4.1 & 0.0 & 0.0 & 3.5 & trace & 0.4 & trace \\
\hline D-mannose (Man) & 25.3 & 11.3 & 2.3 & $0.0^{d}$ & 0.2 & 2.2 & 0.1 & 0.0 & 1.7 \\
\hline D-arabinose (D-Ara) & 32.1 & 2.2 & 1.8 & $23.0^{\mathrm{a}}$ & 0.1 & 3.0 & $0.0^{d}$ & 0.0 & 0.0 \\
\hline L-arabinose (L-Ara) & 27.0 & 12.3 & 6.1 & 0.0 & 0.1 & 1.7 & 0.1 & 0.0 & 3.8 \\
\hline D-xylose $(X y l)$ & 34.6 & 6.4 & 2.1 & $0.0^{\mathrm{d}}$ & 0.0 & 3.3 & 0.1 & 0.0 & 3.5 \\
\hline D-fucose (D-Fuc) & 36.6 & 11.0 & 3.8 & 0.9 & 2.2 & 9.1 & 0.0 & 0.0 & 0.0 \\
\hline L-fucose (L-Fuc) & 33.5 & 8.0 & 6.7 & 0.3 & $N^{e}$ & 7.1 & 0.0 & 0.0 & trace \\
\hline
\end{tabular}

The unit of the metabolites is $\mathrm{mM} / \mathrm{OD}_{600}$.

a The concentrations of major metabolites were determined using HPLC.

${ }^{b}$ Estimated concentrations were based on manual spectral peak deconvolution, rather than by automated (Chenomx) method.

c Acetoin could be quantified by the Chenomx 7.61 software, not the Chenomx 7.51 version.

d The metabolites could be detected in the continuous cultures, not in the batch cultures.

e Concentration could not be accurately determined. 

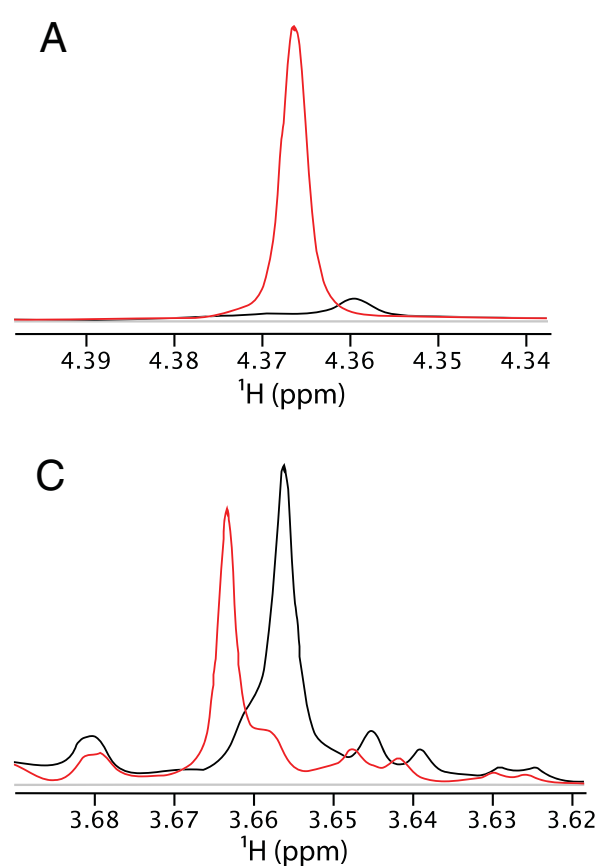


Figure 1 Spectra illustrating selected identified compounds in cell extracts and supernatants. Black line represents extract spectral line, red represents supernatant spectral line. Panel A (mannose substrate) shows peak for hydroxyacetone (singlet at 4.368ppm, not in Chenomx database) present in supernatant (red) and not in extract (black). Panel B (L-arabinose substrate) shows methyl doublet for acetoin (1.368 ppm) present in supernatant (red) but not cell extract (black). Panel C (D-arabinose substrate) shows ethylene glycol present in both supernatant (red) and extract (black), and Panel D (L-fucose substrate) shows propylene glycol present in both supernatant (red) and extract (black).

then comparing the areas to peaks of known concentration. Compound identifications were also confirmed by comparison with prepared standards of individual compounds. In particular, the 2,3-butanediol was shown by comparison to standards to be (2R,3R) and/or (2S,3S)-butanediol, rather than the meso (2R,3S)-butanediol diastereomer. We did not assay optical activity of the butanediol to determine what proportions of the two enantiomers were produced. Bacterial production of all three stereoisomers has been reported in various microorganisms [16]. C. saccharolyticus is predicted to be capable of producing 1,2-propanediol from fermentation of L-fucose on the basis of the presence of a predicted lactaldehyde reductase gene [4]; our results confirm this prediction. In addition to these major products, we also observed production of the amino acids alanine and glycine. Although alanine and glycine are present in the culture medium, concentrations of both amino acids in the supernatant were

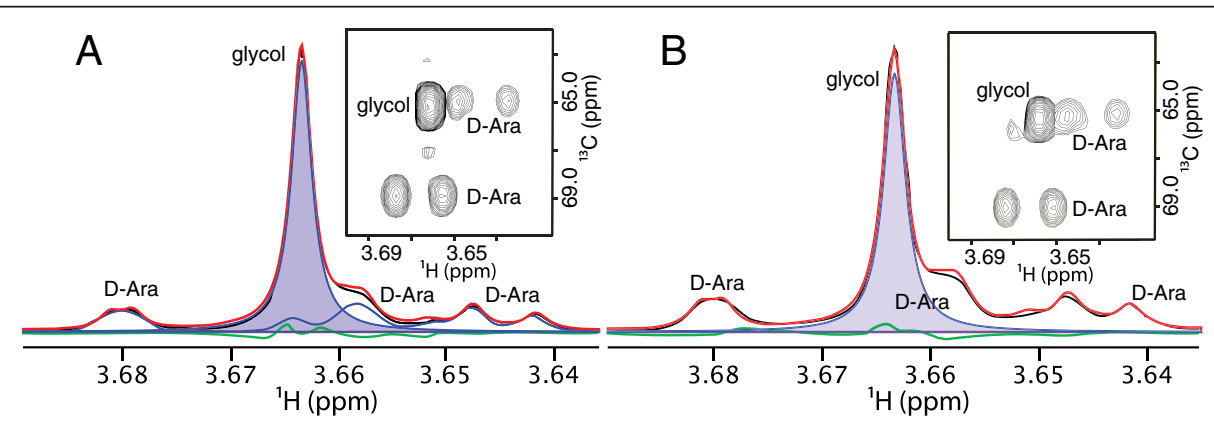

Figure 2 Spectra illustrating identification and confirmation of ethylene glycol in cultures utilizing D-arabinose as substrate. In both panels, the black line indicates the original experimental data, the red line indicates the sum of the fits of individual spectral components (arabinose and ethylene glycol) identified by Chenomx, and the green line indicates the difference spectrum of the experimental and sum-fit spectra. Panel A shows the spectrum of the uninoculated media with (black line) and without (unshaded blue line) ethylene glycol, and the shaded blue area represents the fit of the ethylene glycol peak after addition to media; the inset shows 2-D HSQC confirming the peak assignments. Panel B shows ethylene glycol present in the culture supernatant of C. saccharolyticus grown on D-arabinose using the same color scheme, absent the uninoculated media spectrum used in panel $\mathbf{A}$; the inset shows the 2-D HSQC spectrum. 

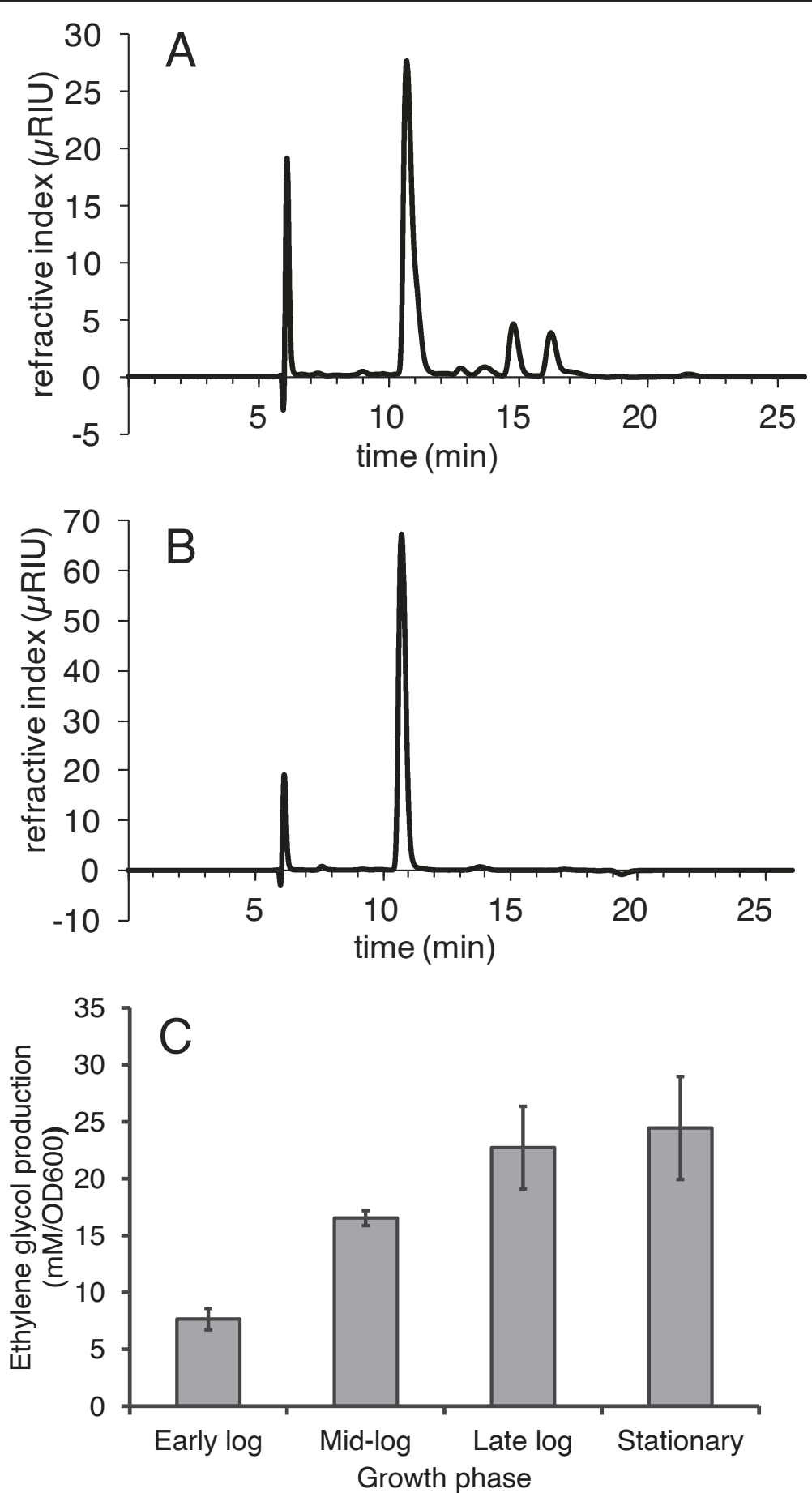

Figure $3 \mathrm{HPLC}$ analysis of ethylene glycol in culture media and ethylene glycol production in different growth phases. Panel A: a representative chromatogram of medium composition obtained from the C. saccharolyticus DSM 8903 strain grown in BA medium supplemented with $10 \mathrm{~g} / \mathrm{L}$ D-arabinose. D-arabinose was eluted at $10.70 \mathrm{~min}$, lactate at $12.77 \mathrm{~min}$, glycerol at $13.69 \mathrm{~min}$, acetate at 14.81 min, ethylene glycol at $16.25 \mathrm{~min}$, and ethanol at $21.50 \mathrm{~min}$. Panel B: BA medium supplemented with $10 \mathrm{~g} / \mathrm{L}$ D-arabinose. Panel C: C. saccharolyticus produces the highest concentrations of ethylene glycol during the late log phase. Values for ethylene glycol concentrations in culture media are from three independent experiments $(n=3)$. Error bars represent standard deviations from the means. 


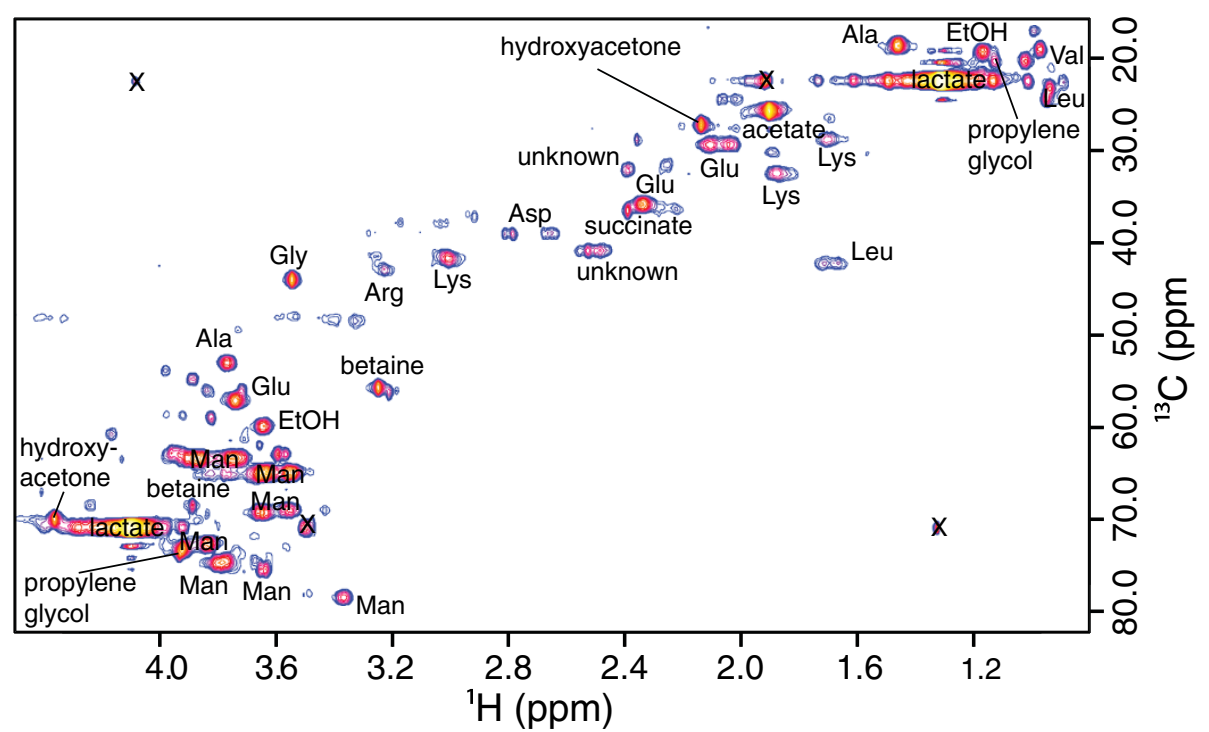

Figure 4 2-D ${ }^{1} \mathrm{H}^{13} \mathrm{C} \mathrm{HSQC}$ of supernatant from culture grown on D-mannose, annotated with compound identifications based on chemical shifts. Standard three letter amino acid and monosaccharide abbreviations are used. Artifact peaks are marked with X. Two unassigned peaks are indicated.

significantly greater compared to their concentrations in the uninoculated growth medium. Yeast extract in the growth medium provides amino acids; alanine was determined to be ca. $0.6 \mathrm{mM}$ and glycine was ca. $0.3 \mathrm{mM}$. Moreover, ${ }^{13} \mathrm{C}$ enrichment in alanine in the supernantant arising from $\left[1-{ }^{13} \mathrm{C}\right]$-glucose in cultures supplemented with this labeled substrate proves that significant alanine production and secretion occurred during these culture growths. None of the major fermentation products (Table 2) were defined components of the growth medium, and we confirmed that none were introduced to the medium by addition of yeast extract.

In summary, C. saccharolyticus was grown on BA media supplemented with different monosaccharide substrates to develop preliminary metabolite profiles for metabolic reconstruction and identify unknown metabolites. These screening experiments revealed several fermentation products that to our knowledge had not been observed previously in C. saccharolyticus: ethylene glycol, 2,3butanediol (RR and/or SS but not meso stereoisomers), acetoin (likely the precursor to 2,3-butanediol), and hydroxyacetone. Of these, ethylene glycol was the most abundant (ca. $23 \mathrm{mM}$ per $\mathrm{OD}_{600}$ in growth on $10 \mathrm{~g} / \mathrm{L}$ $\mathrm{D}$-arabinose during late $\log$ phase) in the culture supernatant. Formation of ethylene glycol, acetoin, and 2,3-butanediol in particular are likely not to be byproducts of non-fermentative processes, rather they are almost certainly the products of fermentative reduction of more oxidized precursors. While ethylene glycol is uncommon, acetoin and 2,3-butanediol are well-known fermentation products in some bacteria [16,17]. However, we were not able to immediately identify a candidate
C. saccharolyticus gene for acetoin formation (presumably, acetolactate decarboxylase), though several candidate acetoin dehydrogenases (Csac_2718, 0357, 0868, or 1605) that could reduce acetoin to 2,3-butanediol have been identified in the genome [4].

C. saccharolyticus cells grew poorly in BA medium supplemented with $1 \%$ glucose without yeast extract. The optical density at a wavelength of $600 \mathrm{~nm}\left(\mathrm{OD}_{600}\right)$ is 0.069 after $48 \mathrm{hr}$ incubation at $65^{\circ} \mathrm{C} ; \mathrm{OD}_{600}$ of cell culture grown in BA medium supplemented with $1 \%$ glucose and $0.2 \%$ yeast extract is 0.283 after $48 \mathrm{hr}$ incubation at $65^{\circ} \mathrm{C}$ based on two independent experiments. Therefore, a richer medium, BA medium supplemented with $0.2 \%$ yeast extract was utilized.

\section{D-arabinose fermentation}

In cells grown on D-arabinose, ethylene glycol was a major product, produced at roughly comparable levels to acetate. Ethylene glycol was not observed in significant quantities as a product of growth on any other substrate used in this study, including L-arabinose. Ethylene glycol production from fermentative anaerobic carbohydrate metabolism appears to be unusual. The likely precursor would be glycolaldehyde, which could be reduced by an alcohol dehydrogenase coded in the $C$. saccharolyticus genome, such as Csac_0622 [4]. The catabolic route of $\mathrm{D}$-arabinose as predicted from the genome [4] does not provide a straightforward route to glycolaldehyde via the non-oxidative pentose phosphate pathway. Indeed, the predicted pathway for $\mathrm{D}$-arabinose catabolism via D-ribulose does not identify a candidate gene for D-ribulokinase that would yield D-ribulose-5-phosphate, 
the precursor to D-xylulose-5-phosphate and/or D-ribose5-phosphate [4]. Furthermore, growth on D-xylose, which is also metabolized via the non-oxidative pentose phosphate pathway and would be expected to yield D-xylulose-5-phosphate, produces only very low levels (less than $200 \mu \mathrm{M}$ ) of ethylene glycol. Instead, a proposed direct route to ethylene glycol from D-arabinose that has been demonstrated in E. coli mutants utilizes the L-fucose pathway, a pathway which appears also to be present in C. saccharolyticus [18]. Indeed, L-fucose isomerase (Csac_1339) is annotated as D-arabinose isomerase [4] and the purified recombinant protein has activity with D-arabinose substrate [19]. In this proposed route, catabolism of the pentose $\mathrm{D}$-arabinose by the (hexose utilizing) L-fucose pathway, could produce two-carbon glycolaldehyde in place of three-carbon lactaldehyde, and the glycoaldehyde could then be reduced to ethylene glycol. The stereochemical configurations at $\mathrm{C} 2, \mathrm{C} 3$, and $\mathrm{C} 4$ in $\mathrm{D}$-arabinose and $\mathrm{L}$-fucose are identical, as has previously been noted [18,19], and the cyclic pyranose form of D-arabinose is identical to that of L-fucose with the exception of the C6 methyl group in L-fucose which is replaced with a hydrogen atom in D-arabinose.

\section{D-xylose and L-arabinose fermentation}

The mixture of fermentation products resulting from growth on D-xylose was somewhat similar to that from growth on L-arabinose. Small amounts of ethanol and glycerol were produced from growth on D-xylose and Larabinose as well (Table 2). These similarities may indicate that D-xylose and L-arabinose utilization pathways merge at D-xylulose-5-phosphate. No 2,3-butanediol was produced in D-xylose or L-arabinose fermentation; however, acetoin and hydroxyacetone were observed in minor amounts in cultures grown on L-arabinose (see Table 2).

\section{D-mannose fermentation}

Growth of C. saccharolyticus on D-mannose, the C-2 epimer of glucose, produced more lactate compared to growth on glucose (Table 2). More lactate production in cultures grown on D-mannose versus that in D-glucose grown cultures $(\sim 8$ fold $)$ is not easily explained. Willquist and van Niel utilized kinetic analysis to determine that $C$. saccharolyticus lactate dehydrogenase (LDH) activity is regulated through competitive inhibition by pyrophosphate and NAD and allosteric activation by fructose-1,6-bisphosphate, ATP and ADP; the authors furthermore concluded that activation of LDH by ATP indicated that C. saccharolyticus utilizes $\mathrm{LDH}$ as a means to adjust ATP and NADH production [8]. Perhaps notable is the observation of VanFossen et al. that substantial differences ( $\geq 2$-fold change in abundance in 353 ORFs) in the C. saccharolyticus transcriptome were seen when cells utilizing either glucose or mannose were compared, while far fewer differences were seen when cells utilizing galactose or glucose were compared (32 ORFs with $\geq 2$-fold change) [5].

Hydroxyacetone was identified in mannose-fed cultures as a minor (approximately $1.7 \mathrm{mM}$ per $\mathrm{OD}_{600}$ ) product. This product was also observed in L-arabinose and D-xylose cultures (see Table 2). It is not clear what hydroxyacetone production implies about D-mannose, L-arabinose and D-xylose metabolism in C. saccharolyticus, though it could be the result of nonspecific enzymatic activity on substrates such as propylene glycol or methylglyoxal. In methanogenic archaea (Methanococcus jannaschii), reduction of methylglyoxal was shown to produce both lactaldehyde and hydroxyacetone [20]. Methylglyoxal is a central metabolite in the synthesis of aromatic amino acids in M. jannaschii. Alternatively, hydroxyacetone may be produced in $M$. jannaschii by condensation of pyruvate with formaldehyde with loss of $\mathrm{CO}_{2}$ [20] though this route is unlikely in $C$. saccharolyticus.

Although non-specific enzyme activity may account for production of metabolites such as hydroxyacetone and acetoin, if these compounds are products of nonspecific reactions on common metabolic intermediate precursors, we might expect to see them across all conditions. Since hydroxyacetone was identified from D-mannose, L-arabinose, and D-xylose cultures and acetoin was identified from L-arabinose, D-arabinose, D-glucose, and D-xylose cultures, it suggests that these metabolites are products of specific enzyme reactions on specific substrates.

\section{D-glucose fermentation}

For cultures grown on glucose, ethanol (around $4.1 \mathrm{mM}$ per $\mathrm{OD}_{600}$ ) and lactate (around $1.4 \mathrm{mM}$ per $\mathrm{OD}_{600}$ ) were present along with the most abundant metabolite, acetate (around $37 \mathrm{mM}$ per $\mathrm{OD}_{600}$ ). The novel metabolites 2,3-butanediol (2R,3R and/or 2S,3S stereoisomers) and acetoin were present at lower concentrations in the culture supernatant. We did not observe diacetyl, a possible precursor to acetoin through a non-enzymatic oxidative decarboxylation of acetolactate that is unlikely in anaerobic conditions. Butanediol fermentation is common in the Gammaproteobacteria and is known in some Firmicutes genera, both in the Clostridia and Bacilli classes, but has not been reported in C. saccharolyticus. Indeed, Klebsiella pneumoniae and Bacillus polymyxa have been discussed as potential industrial scale producers of 2,3-butanediol, utilizing a mixed acid fermentation pathway whose other end-products include ethanol, acetate, lactate, formate, and succinate [16]. In these organisms, formation of 2,3-butanediol begins with condensation of two pyruvates by acetolactate synthetase to yield acetolactate and 
$\mathrm{CO}_{2}$ [21,22]. Acetohydroxyacid synthetases are common, due to their role in biosynthesis of L-valine, L-leucine and L-isoleucine. The C. saccharolyticus genome has genes for two such enzymes (Csac_0837 and Csac_1142/1143) annotated as acetolactate synthases. Under anaerobic conditions, decarboxylation of acetolactate by acetolactate decarboxylase produces acetoin [22]. Acetoin, typically the precursor of 2,3-butanediol, can be reduced in a reversible reaction catalyzed by acetoin reductase, which also catalyzes the irreversible reduction of diacetyl to acetoin [16]. However, we could not identify an acetolactate decarboxylase in the C. saccharolyticus genome.

An alternate route to 2,3-butanediol from acetoin via diacetyl and acetylacetoin has also been suggested in which diacetyl is acetylated and reduced to yield acetylbutanediol which is then hydrolyzed to 2,3-butanediol and acetate [23]. We cannot rule out that. C. saccharolyticus may use this alternate route or some variation of the typical route to 2,3-butanediol, or it may have a novel or atypical acetoin reductase that cannot be identified by sequence comparisons. In any case, identification of the genes essential for 2,3-butanediol formation and determination of the stereochemistry of this mechanism will be important goals going forward.

\section{D- and L-fucose metabolism}

A limited batch culture investigation of growth on the deoxyhexoses L-fucose and D-fucose was conducted to confirm genome-based predictions about their metabolism [4], particularly as it relates to D-arabinose metabolism (vide infra). These substrates did not support robust growth in our lab (highest $\mathrm{OD}_{600}$ of L-fucose culture is 0.10 ; highest $\mathrm{OD}_{600}$ of $\mathrm{D}$-fucose culture is 0.08 according to three independent batch cultures), though growth on this substrate has been reported previously [1] and was predicted on the basis of the presence of two genes in the C. saccharolyticus genome, Csac_1340 and Csac_1339 that code for putative $\alpha$-L-fucosidase and L-fucose isomerase enzymes. The predicted pathway results in 1,2-propanediol formation, in agreement with our observations. If the same pathway were used to support metabolism of the pentose D-arabinose, ethylene glycol would be the expected fermentation product. This expectation was confirmed in our results, and indeed appears to be more facile than conversion of L-fucose to 1,2-propanediol. As with L-fucose, growth on D-fucose was slow, requiring $48 \mathrm{hr}$ to reach an OD of approximately 0.08 . The route of D-fucose utilization in C. saccharolyticus is currently under investigation.

\section{Conclusions}

Our approach using $1-\mathrm{D}{ }^{1} \mathrm{H}$ and ${ }^{13} \mathrm{C}$ NMR spectroscopy to characterize product mixtures from monosaccharide fermentation by $C$. saccharolyticus identified numerous components in culture supernatants that were not present in the growth medium prior to inoculation. Components that could not be assigned from 1-D spectra because they were not present in our spectral databases were assigned and identified with 2-D NMR experiments and confirmed by comparison to authentic standards. This approach has particular advantages over other methods of analyzing products of microbial cell culture. Minimal sample manipulation is required, no derivatization is necessary, and information useful for identification of novel metabolites is obtained. The main disadvantage, of course, is the inherent low sensitivity of NMR spectroscopy, such that minor metabolite components that might be of interest may not be observable.

Together, the results suggest that C. saccharolyticus, already of substantial interest due to its potential for biological ethanol and hydrogen production, has further metabolic potential for production of the higher molecular weight compounds acetoin and 2,3-butanediol, as well as glycerol, hydroxyacetone, and the uncommon fermentation product ethylene glycol. In addition to these alcohols, production of acetate and lactate was observed. Formation of reduced, non-acidic, fermentation products may be a built-in mechanism for bacteria to mitigate excess acetate or lactate formation, which could decrease the $\mathrm{pH}$ of the growth medium to a point that may not be beneficial to the organism. Conceivably, metabolic pathways could be engineered to divert carbon away from two-carbon products such as ethanol and acetate and towards reduced three and four carbon products such as glycerol or butanediol. The findings presented here seem to suggest C. saccharolyticus has multiple routes available by which this strategy could be implemented. Furthermore our finding that $C$. saccharolyticus produces substantial amounts of ethylene glycol during growth on D-arabinose, apparently from glycolaldehye by the L-fucose pathway (which perhaps should be called the L-fucose/D-arabinose pathway) adds this reduced fermentation product that may be of interest in industrial biotechnology as a product of lignocellulosic biomass.

\section{Materials and methods \\ Reagents}

Components of the growth medium were obtained from Sigma (St. Louis, MO) and used without further purification. Carbon-13 labeled glucose $\left(\left[1-{ }^{13} \mathrm{C}\right]-\mathrm{D}\right.$-glucose $)$ was obtained from Cambridge Isotope Laboratories (Andover, MA).

\section{Bacterial strain and growth conditions}

C. saccharolyticus DSM 8903 was obtained from the Deutsche Sammlung von Mikroorganismen und Zellkulturen (DSMZ). C. saccharolyticus was grown in the anaerobic BA medium. The BA medium composition has been described previously $[24,25]$. BA medium contains 
$18.7 \mathrm{mM} \mathrm{NH}_{4} \mathrm{Cl}, 1.71 \mathrm{mM} \mathrm{NaCl}, 0.5 \mathrm{mM} \mathrm{MgCl}_{2}, 0.34$ $\mathrm{mM} \mathrm{CaCl} 2,1.8 \mathrm{mM} \mathrm{K} \mathrm{HPO}_{4}, 2 \mu \mathrm{M}$ resazurin, $0.81 \mu \mathrm{M}$ boric acid, $0.37 \mu \mathrm{M} \mathrm{ZnCl}, 0.22 \mu \mathrm{M} \mathrm{CuCl}_{2}, 0.25 \mu \mathrm{M}$ $\mathrm{MnCl}_{2}, 0.040 \mu \mathrm{M}(\mathrm{NH} 4)_{6} \mathrm{Mo}_{7} \mathrm{O}_{24}, 0.37 \mu \mathrm{M} \mathrm{AlCl}, 0.21 \mu \mathrm{M}$ $\mathrm{CoCl}_{2}, 0.39 \mu \mathrm{M} \mathrm{NiCl}, 1.34 \mu \mathrm{M}$ EDTA, $0.25 \mu \mathrm{M} \mathrm{Na}_{2} \mathrm{SeO}_{3}$, and $9.83 \mu \mathrm{M} \mathrm{FeCl}_{2}$. The vitamin solution and cysteine used previously in BA medium were omitted, and instead the medium was supplemented with $2 \mathrm{~g} / \mathrm{L}$ yeast extract and the appropriate monosaccharide substrate at a concentration of $10 \mathrm{~g} / \mathrm{L}$. Media were made anaerobic by flushing with $\mathrm{N}_{2} / \mathrm{CO}_{2}(80 / 20$, v/v).

To compare the growth and the metabolite levels across the different monosaccharides tested, C. saccharolyticus was grown on D-glucose, D-mannose, D-xylose, Larabinose, D-arabinose, L-fucose, and D-fucose in batch cultures. The growth was further tested in continuous culture with L-arabinose, D-arabinose, D-mannose and Dxylose as substrate. Batch cultivation experiments were performed with a culture volume of $20 \mathrm{ml}$ in an airtight flask at $65^{\circ} \mathrm{C}$. Continuous cultivation was performed $60^{\circ} \mathrm{C}$ in an INFORS HT Multifors (Basel, Switzerland) bench top bioreactor at a constant working volume of $0.5 \mathrm{~L}$ with stirring at $100 \mathrm{rpm}$. The $\mathrm{pH}$ was controlled at 7.0 by automatic addition of $\mathrm{NaOH}$ to the vessel. Fresh media was added at a rate of $0.12 \mathrm{ml} / \mathrm{min}$. The software Iris V5 was used to control the bioreactor and analyze and archive the data. The BA medium in the vessel of the bioreactor was inoculated with $1 \%(\mathrm{v} / \mathrm{v})$ of seed culture in the exponential growth phase. A culture was considered to have reached steady state when the bacterial culture remained at a constant optical density (OD) at wavelength of $600 \mathrm{~nm}$, acetate or lactate production rates remained constant (monitored by HPLC), and at least four volume changes (2 L) had occurred. Samples were collected at steady states for the determination of intracellular and extracellular metabolites.

\section{Sample preparation for NMR analysis}

To prepare samples of extracellular metabolites for identification and quantification by NMR spectroscopy, $1 \mathrm{ml}$ volumes were removed from the culture and centrifuged at $14000 \mathrm{rpm}(20,800 \mathrm{rcf})$ for 5 minutes at $4^{\circ} \mathrm{C}$. The resulting supernatant samples were not filtered. For each sample, a supernatant volume of $540 \mu \mathrm{l}$ was mixed with $60 \mu \mathrm{l}$ of $\mathrm{D}_{2} \mathrm{O}$ solution containing $5 \mathrm{mM}$ DSS-d6 (2,2 dimethyl 2-silapentane-d6 5-sulfonate, sodium salt) for chemical shift referencing and $0.2 \%(\mathrm{w} / \mathrm{v})$ sodium azide as a microbiocide. To determine intracellular metabolites, a sample of $10 \mathrm{ml}$ was harvested and centrifuged. The cell pellet was mixed with ice-cold chloroform/methanol $(2: 1, \mathrm{v} / \mathrm{v})$ solution and subjected to three cycles of freeze/ thaw using liquid nitrogen. Following centrifugation, the sample separated into three layers (aqueous, cell debris, and organic). The entire upper layer (aqueous $\mathrm{MeOH}$ ) was removed and evaporated under vacuum centrifugation in a Savant SpeedVac concentrator (Thermo Fisher). For each sample, the resultant dried extract was mixed with $300 \mu$ of $\mathrm{D}_{2} \mathrm{O}$ solution containing $0.5 \mathrm{mM}$ DSS-d6 for chemical shift referencing and $0.2 \%(\mathrm{w} / \mathrm{v})$ sodium azide as a microbiocide.

\section{NMR spectroscopy}

Extracellular metabolite samples were placed in $5 \mathrm{~mm}$ 535-PP NMR tubes (Wilmad), and intracellular samples were placed in $5 \mathrm{~mm}$ Shigemi NMR tubes. All NMR spectra were collected at $25^{\circ} \mathrm{C}$ on a $600 \mathrm{MHz}$ Agilent (Varian) NMR System equipped with a salt-tolerant 5 mm HCN coldprobe with cold carbon preamplifier for higher sensitivity in ${ }^{13} \mathrm{C}$-observe experiments. Samples contained 0.5 mM DSS-d6 for chemical shift referencing and as an internal standard for quantification. For Chenomx analysis, 1-D NOESY spectra were collected using the Varian tnnoesy pulse sequence with $12 \mathrm{ppm}$ spectral width, acquisition time of 4 seconds, mixing time of 100 milliseconds, relaxation delay of $1 \mathrm{~s}$, and 128 scans. Direct observe $1-\mathrm{D}{ }^{13} \mathrm{C}$ spectra were collected using a $224 \mathrm{ppm}$ spectral width, a tip angle of $45^{\circ}$, a relaxation delay of 3 seconds, and WALTZ proton decoupling during the acquisition time of 1.3 seconds. Two-dimensional ${ }^{1} \mathrm{H}_{-}{ }^{1} \mathrm{H}$ magnitude COSY and ${ }^{1} \mathrm{H}_{-}{ }^{13} \mathrm{C}$ HSQC and HMBC experiments were collected using Varian gCOSY, gHSQC, and gHMBC pulse sequences with ${ }^{1} \mathrm{H}$ spectral width of $12 \mathrm{ppm}$ and ${ }^{13} \mathrm{C}$ spectral widths of $170 \mathrm{ppm}$ (HSQC) or $240 \mathrm{ppm}$ (HMBC), with an acquisition time of 200 milliseconds (1445 complex points), 128 complex points in the indirect dimension for HSQC and HMBC and 512 for COSY experiments, 128 transients, 1s recycle delay, and adiabatic WURST decoupling (Varian W40_Coldprobe) during acquisition in the HSQC experiment.

\section{NMR data analysis}

One-dimensional ${ }^{1} \mathrm{H}$ spectra were processed and analyzed with Chenomx software version 7.61 (Edmonton, AB) with $0.5 \mathrm{~Hz}$ line broadening and automatic baseline correction. Quantification of 1-D spectra relied on comparison of peak areas in compound peak clusters to the concentration standard of $0.5 \mathrm{mM}$ DSS-d6 (2,2 dimethyl 2-silapentane-d6 5 -sulfonate, sodium salt) in the Chenomx database. For analysis of extracellular (secreted) metabolites in particular, essentially no manipulation of the sample is required aside from addition of a small amount of reference solvent containing $\mathrm{D}_{2} \mathrm{O}$ for locking the spectrometer and DSS-d6 for referencing the spectra. Spectral features not assigned by Chenomx were further characterized using 2-D NMR experiments. Compounds were identified by de novo assignment of spin systems in ${ }^{1} \mathrm{H}_{-}{ }^{13} \mathrm{C}$ HSQC and $\mathrm{HMBC}$, and ${ }^{1} \mathrm{H}-{ }^{1} \mathrm{H}$ magnitude COSY experiments, and 
identifications were confirmed by spectral comparison to authentic compounds. Concentrations of novel metabolites were estimated using spectral deconvolution routines within the spectrometer software (Varian/Agilent VnmrJ). Two-dimensional NMR data was processed using Felix (FelixNMR, Inc). The 2-D heteronuclear experiments were processed with time-domain convolution of the water resonance followed by apodization with a 90 degree shifted sinebell window matched to the entire FID and zero-filling to twice the number of real points. The same apodization and zero-filling were applied to the indirect dimension after linear prediction of 30\% more real points. Magnitude COSY spectra were processed in both dimensions with 10 degree-shifted squared sinebell apodization and zerofilling following time-domain solvent deconvolution of the acquired data.

\section{HPLC analysis}

To analyze extracellular metabolites using HPLC, $1 \mathrm{~mL}$ samples were removed from the $C$. saccharolyticus DSM 8903 culture and centrifuged at $14000 \mathrm{rpm}(20,800 \mathrm{rcf})$ for 5 minutes at $4^{\circ} \mathrm{C}$. The resulting supernatants were filtered before HPLC analysis. Samples were analyzed using an Ultimate 3000 HPLC system (Dionex) consisting of a pump, an autosampler and a column compartment. The column was a $300 \mathrm{~mm} \times 7.8 \mathrm{~mm}$ Aminex HPX-87H column (Bio-Rad) and the column temperature was $60^{\circ} \mathrm{C}$. The eluent was $4 \mathrm{mM}$ sulfuric acid solution. The flow rate was maintained at $0.6 \mathrm{ml} / \mathrm{min}$. The HPLC system was equipped with a refractive index detector (RI-101, Shodex). Chromeleon 7 software was used to integrate the peaks and quantify the metabolites.

\section{Bioinformatics}

Candidate genes for acetoin and butanediol production were searched using PSI-BLAST to find sequence homologues of annotated acetolactate synthase, acetolactate decarboxylase, and acetoin dehydrogenase genes from other bacteria.

\section{Additional file}

\section{Additional file 1: Metabolite concentrations determined using} proton NMR spectroscopy $(\mu \mathrm{M})$.

\section{Abbreviations}

COSY: COrrelation SpectroscopY; ED: Entner-Doudoroff; EM: EmbdenMeyerhof; HSQC: Heteronuclear Single Quantum Coherence spectroscopy; HMBC: Heteronuclear Multiple Bond Correlation spectroscopy; NMR: Nuclear Magnetic Resonance.

\section{Competing interests}

The authors declare they have no competing interests.

\section{Authors' contributions}

$\mathrm{NI}$ designed the NMR studies, collected and analyzed NMR data, and wrote the manuscript. JX designed and conducted batch and continuous culture studies, prepared samples, collected HPLC data, and revised the manuscript. JR participated in continuous culture studies and sample preparation. JC analyzed NMR data and wrote the manuscript. BK led design of the $C$ saccharolyticus studies and revised the manuscript. All authors have read and approved the manuscript.

\section{Acknowledgements}

We gratefully acknowledge financial support from the Washington States STAR researcher program given to Prof. Ahring 2008-2010, from the Danish Strategic Research Council, which supported growth of C. saccharolyticus cultures under their Biorefinery project, and from the Environmental Molecular Sciences Laboratory (EMSL) Intramural program, which funded the development and implementation of NMR methods for this project. The NMR spectroscopy portion of the research was performed using EMSL, a national scientific user facility sponsored by the Department of Energy's Office of Biological and Environmental Research and located at Pacific Northwest National Laboratory.

We are grateful to Weiqun Zhong and Jens Iversen (WSU) and to Paul Majors (EMSL) for their advice and assistance.

\section{Author details}

${ }^{1}$ Environmental Molecular Sciences Laboratory, Pacific Northwest National Laboratory, Richland, WA 99354, USA. ${ }^{2}$ Fundamental and Computational Sciences Directorate, Pacific Northwest National Laboratory, PO Box 999, MSIN: K8-98, Richland, WA 99352, USA. ${ }^{3}$ Center for Bioproducts and Bioenergy, Washington State University, 2710 Crimson Way, Richland, WA 99354, USA.

Received: 6 December 2012 Accepted: 7 March 2013

Published: 3 April 2013

\section{References}

1. Rainey FA, Donnison AM, Janssen PH, Saul D, Rodrigo A, Bergquist PL, Daniel RM, Stackebrandt E, Morgan HW: Description of Caldicellulosiruptor saccharolyticus gen. nov., sp. nov: an obligately anaerobic, extremely thermophilic, cellulolytic bacterium. FEMS Microbiol Lett 1994, 120(3):263-266.

2. de Vrije T, Bakker RR, Budde MA, Lai MH, Mars AE, Claassen PA: Efficient hydrogen production from the lignocellulosic energy crop Miscanthus by the extreme thermophilic bacteria Caldicellulosiruptor saccharolyticus and Thermotoga neapolitana. Biotechnol Biofuels 2009, 2(1):12.

3. de Vrije T, Mars AE, Budde MA, Lai MH, Dijkema C, de Waard P, Claassen PA: Glycolytic pathway and hydrogen yield studies of the extreme thermophile Caldicellulosiruptor saccharolyticus. Appl Microbiol Biotechnol 2007, 74(6):1358-1367.

4. van de Werken $H J$, Verhaart MR, VanFossen AL, Willquist $K$, Lewis DL, Nichols JD, Goorissen HP, Mongodin EF, Nelson KE, van Niel EW, et al: Hydrogenomics of the extremely thermophilic bacterium Caldicellulosiruptor saccharolyticus. Appl Environ Microbiol 2008, 74(21):6720-6729.

5. VanFossen AL, Verhaart MR, Kengen SM, Kelly RM: Carbohydrate utilization patterns for the extremely thermophilic bacterium Caldicellulosiruptor saccharolyticus reveal broad growth substrate preferences. Appl Environ Microbiol 2009, 75(24):7718-7724.

6. Willquist K, Zeidan AA, van Niel EW: Physiological characteristics of the extreme thermophile Caldicellulosiruptor saccharolyticus: an efficient hydrogen cell factory. Microb Cell Fact 2010, 9:89.

7. Maki M, Leung KT, Qin W: The prospects of cellulase-producing bacteria for the bioconversion of lignocellulosic biomass. Int J Bio/ Sci 2009, 5(5):500-516.

8. Willquist $\mathrm{K}$, van Niel EW: Lactate formation in Caldicellulosiruptor saccharolyticus is regulated by the energy carriers pyrophosphate and ATP. Metab Eng 2010, 12(3):282-290.

9. Dieterle F, Riefke B, Schlotterbeck G, Ross A, Senn H, Amberg A: NMR and MS methods for metabonomics. Meth Mol Biol 2011, 691:385-415.

10. Weljie AM, Newton J, Jirik FR, Vogel HJ: Evaluating low-intensity unknown signals in quantitative proton NMR mixture analysis. Anal Chem 2008, 80(23):8956-8965.

11. Weljie AM, Newton J, Mercier P, Carlson E, Slupsky CM: Targeted profiling: quantitative analysis of $1 \mathrm{H}$ NMR metabolomics data. Anal Chem 2006, 78(13):4430-4442

12. Behrends V, Bundy JG, Williams HD: Differences in strategies to combat osmotic stress in Burkholderia cenocepacia elucidated by NMR-based metabolic profiling. Lett App/ Microbiol 2011, 52(6):619-625. 
13. Booth SC, Workentine ML, Wen J, Shaykhutdinov R, Vogel HJ, Ceri H, Turner RJ, Weljie AM: Differences in metabolism between the biofilm and planktonic response to metal stress. J Proteome Res 2011, 10(7):3190-3199.

14. Tredwell GD, Edwards-Jones B, Leak DJ, Bundy JG: The development of metabolomic sampling procedures for Pichia pastoris, and baseline metabolome data. PLoS One 2011, 6(1):e16286.

15. Kim YM, Lee SE, Park BS, Son MK, Jung YM, Yang SO, Choi HK, Hur SH, Yum $\mathrm{JH}$ : Proteomic analysis on acetate metabolism in Citrobacter sp. BL-4. Int J Biol Sci 2012, 8(1):66-78.

16. Syu MJ: Biological production of 2,3-butanediol. Appl Microbiol Biotechnol 2001, 55(1):10-18.

17. Garg SK, Jain A: Fermentative production of 2,3-butanediol - a review. Bioresour Technol 1995, 51(2-3):103-109.

18. LeBlanc DJ, Mortlock RP: Metabolism of D-arabinose: a new pathway in Escherichia coli. J Bacteriol 1971, 106(1):90-96.

19. Ju YH, Oh DK: Characterization of a recombinant L-fucose isomerase from Caldicellulosiruptor saccharolyticus that isomerizes L-fucose, Darabinose, D-altrose, and L-galactose. Biotechnol Lett 2010, 32(2):299-304.

20. White RH: Biochemical origins of lactaldehyde and hydroxyacetone in Methanocaldococcus jannaschii. Biochemistry 2008, 47(17):5037-5046.

21. Romick TL, Fleming HP: Acetoin production as an indicator of growth and metabolic inhibition of Listeria monocytogenes. J Appl Microbiol 1998, 84(1):18-24.

22. Nielsen DR, Yoon SH, Yuan CJ, Prather KL: Metabolic engineering of acetoin and meso-2, 3-butanediol biosynthesis in E. coli. Biotechnol J 2010 5(3):274-284.

23. Ui S, Hosaka T, Mizutani K, Mimura A: Purification and properties of acetylacetoin synthase from Bacillus sp. YUF-4. Biosci Biotechnol Biochem 1998, 62(4):795-797.

24. Angelidaki I, Petersen SP, Ahring BK: Effects of lipids on thermophilic anaerobic digestion and reduction of lipid inhibition upon addition of bentonite. Appl Microbiol Biotechnol 1990, 33(4):469-472.

25. Sommer P, Georgieva T, Ahring BK: Potential for using thermophilic anaerobic bacteria for bioethanol production from hemicellulose. Biochem Soc Trans 2004, 32(Pt 2):283-289.

doi:10.1186/1754-6834-6-47

Cite this article as: Isern et al: Novel monosaccharide fermentation products in Caldicellulosiruptor saccharolyticus identified using NMR spectroscopy. Biotechnology for Biofuels 2013 6:47.

\section{Submit your next manuscript to BioMed Central and take full advantage of:}

- Convenient online submission

- Thorough peer review

- No space constraints or color figure charges

- Immediate publication on acceptance

- Inclusion in PubMed, CAS, Scopus and Google Scholar

- Research which is freely available for redistribution

Submit your manuscript at www.biomedcentral.com/submit
(O) Biomed Central 